Article

\title{
Broad-Band Transmission Characteristics of Polarizations in Foggy Environments
}

\author{
Tianwei Hu ${ }^{1,2}{ }^{\text {, Fei Shen }}{ }^{1}$, Kaipeng Wang ${ }^{1}$, Kai Guo ${ }^{1, *}{ }^{1}$, Xiao Liu ${ }^{2}$, Feng Wang ${ }^{2}$, Zhiyong Peng ${ }^{3}$, \\ Yuemeng Cui ${ }^{3}$, Rui Sun ${ }^{1} \mathbb{D}$, Zhizhong Ding ${ }^{1}$, Jun Gao ${ }^{1}$ and Zhongyi Guo ${ }^{1,2,3, * \mathbb{D}}$ \\ 1 School of Computer and Information, Hefei University of Technology, Hefei 230009, China; \\ hutianwei528@163.com (T.H.); shenfei@hfut.edu.cn (F.S.); wangkaipeng1206@163.com (K.W.); \\ sunrui@hfut.edu.cn (R.S.); zzding@hfut.edu.cn (Z.D.); gaojun@hfut.edu.cn (J.G.) \\ 2 Anhui Key Laboratory of Polarization Imaging Detection Technology, Hefei 230009, China; \\ liuxiao_0007@163.com (X.L.); wfissky@sina.com (F.W.) \\ 3 Tianjin Jinhang Institute of Technology and Physics, Tianjin 300300, China; foxdox@163.com (Z.P.); \\ cymeng@sina.com (Y.C.) \\ * Correspondence: kai.guo@hfut.edu.cn (K.G.); guozhongyi@hfut.edu.cn (Z.G.)
}

Received: 12 April 2019; Accepted: 20 June 2019; Published: 24 June 2019

\begin{abstract}
Based on the Monte Carlo (MC) algorithm, we simulate the evolutions of different types of the polarized lights in the broad-band range from visible to infrared in foggy environments. Here, we have constructed two scattering systems to simulate the transmission characteristics of the polarized lights: (1) A monodisperse system based on five types of particles with the sizes of $0.5,1.0,2.5,4$, and $5 \mu \mathrm{m}$, respectively; (2) a polydisperse system based on scattering particles with a mean value (size) of $2.0 \mu \mathrm{m}$. Our simulation results show that linearly polarized light (LPL) and circularly polarized light (CPL) exhibit different advantages in different wavelengths and different scattering systems. The polarization maintenances (PM) of the degree of circular polarizations (DoCPs) are better than those of the degree of linear polarizations (DoLPs) for most incident wavelengths. CPL is not superior to LPL in the strong-absorption wavelengths of $3.0 \mu \mathrm{m}, 6.0 \mu \mathrm{m}$, and long infrared. Here, when the wavelength is closer to the particle sizes in a system, the influence on propagating polarizations will be more obvious. However, the difference in the degree of polarization (DoP) between the resulting CPL and LPL is positive at these points, which means the penetrating ability of CPL is superior to that of LPL in these scattering systems. We have also simulated the extinction efficiency $Q_{\text {ext }}$ and the scattering index ratio $Q_{\text {ratio }}$ as functions of both wavelength and particle size for analyzing polarization's transmission characteristics. Our work paves the way of selecting the optimal incident wavelengths and polarizations for concrete scattering systems.
\end{abstract}

Keywords: Monte Carlo (MC) algorithm; degree of polarization (DoP); visibility level; foggy environments

\section{Introduction}

A foggy environment is often formed in low altitude. When travelling in a foggy environment, light will undergo scattering and absorption by the water droplets, during which light intensity information will be severely attenuated, affecting the long-distance transmission efficiency of optical information [1]. Next to its wavelength, amplitude, and phase, polarization is an independent dimension of light which has attracted more and more attention due to the polarization maintenance (PM) of light when it propagates in scattering systems. Jessica C. et al. proposed three methods for simulating polarized light scattering processes based on the Monte Carlo (MC) algorithm [2,3]. Wang L.V. et al. used a time-resolved MC technique to investigate the propagating effects of incident polarization states in turbid media under the wavelength of $543 \mathrm{~nm}$ [4]. Xu M. et al. demonstrated that 
the optimal circularly polarized light (CPL) memory would occur for systems of large-sized particles and small-sized particles with high refractive index (RI) particles [5]. Sun P. et al. experimentally investigated the PM of backscattered CPL and linearly polarized light (LPL) in polystyrene sphere suspensions, and they found that the PM of backscattered LPL was superior to that of backscattered CPL when particles' sizes were smaller than incident wavelength [6]. Schechner Y.Y. et al. conducted polarization imaging experiments in foggy conditions, and they found that polarization imaging could eliminate or weaken the influence of a scattering system compared with traditional intensity imaging, thus improving contrast and the visibility of imaging [7]. Van der Laan J. D. et al. simulated the transmission characteristics of CPL in a foggy environment for improving the detection range [8-10]. $\mathrm{Hu} \mathrm{H}$. et al. used the memory effect of CPL to improve the quality of recovered polarized images [11]. $\mathrm{Li}$ X. et al. proposed a pseudo-polarimetric dehazing method, which only required a single image captured by the detector [12]. Actually, the setting of the target DoP (degree of polarization) is related to specific foggy conditions; therefore, it is necessary to fully explore polarization transmission characteristics in different foggy environments. Liu F. et al. have proposed an active polarization imaging technique based on wavelength selection and the optical correlation theory [13]. Similarly, the exploration of polarization transmission characteristics is also extremely important.

In our previous study, we investigated polarization characteristics under different medium conditions and proposed a new recovery method for the transmitted polarization information [14-20]. Our former works were mainly focused on characteristics in the visible band. Here, we present a quantitative investigation on polarization transmission characteristics under foggy conditions with an extending range of incident wavelengths from the visible to infrared bands. In order to be more exploratory and practical, we have defined the visibility of the medium and simulated both the influences of the transmission distance of light and the sizes of scattering particles on both CPL and LPL propagating performances. Under the same visibility condition $\left(V=-\ln \varepsilon / u_{e}(550 \mathrm{~nm})\right)$ [21-23], the dependence of performance on incident wavelength produces greater polarization sensitivity, and the penetrating performances of CPL are better than those of LPL for systems with specific particle sizes. The results show that our work provides a theoretical basis for the selection of incident wavelength and polarizations for the turbid scattering systems.

\section{Experiments}

The scattering and absorption of water foggy particles not only attenuates light intensity but also changes the polarization states of the incident light, which will be affected by wavelength, particle sizes, and so on. Here, based on the Mie scattering theory, we present a point-to-point MC transport model for the purpose of exploring the transmission characteristics of different polarizations in foggy environments, as depicted in Figure 1. In our model, a large number of photons $\left(10^{6}\right)$ are normally incident into a single homogeneous medium in which photons interact with the scattering particles, and each scattering event is independent with each other. Simultaneously, the scattering photons from different transmission paths are collected by the semi-infinite forward detection plane.

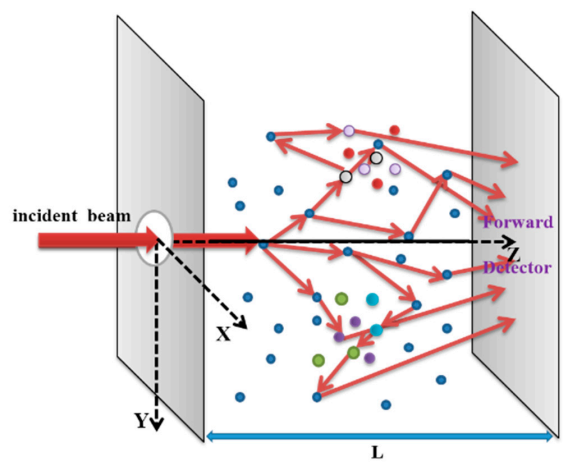

Figure 1. The schematic of the Monte Carlo (MC) transport model. 
Meanwhile, we describe polarization state of incident light by a Stokes-vector $\left(S=[I, Q, U, V]^{T}\right)$, and the process of scattering can be defined by the Stokes-Mueller formalism, as described in equation [24].

$$
\begin{gathered}
\mathbf{S}=\left[\begin{array}{c}
I \\
Q \\
U \\
V
\end{array}\right]=\left[\begin{array}{c}
\left\langle E_{x} E_{x}^{*}+E_{y} E_{y}^{*}\right\rangle \\
\left\langle E_{x} E_{x}^{*}-E_{y} E_{y}^{*}\right\rangle \\
\left\langle E_{x} E_{y}^{*}+E_{y} E_{x}^{*}\right\rangle \\
i\left\langle E_{x} E_{y}^{*}-E_{y} E_{x}^{*}\right\rangle
\end{array}\right]=\left[\begin{array}{c}
I_{x}+I_{y} \\
2 I_{x}-I \\
2 I_{45^{\circ}}-I \\
2 I_{R}-I
\end{array}\right], \\
\mathbf{S}_{0}=\mathbf{M S}_{i}=\mathbf{R}(\varphi)\left[\prod_{k=0}^{k^{\prime}} \mathbf{R}_{k}(\beta) \mathbf{M}_{k}(\theta) \mathbf{R}_{k}(\alpha)\right] \mathbf{S}_{i},
\end{gathered}
$$

where $s_{0}$ and $s_{i}$ represent the scattering and incident Stokes-vector, respectively; $\mathbf{R}$ is the rotation matrix with respect to the reference plane and the scattering plane; $\alpha, \beta$ and $\varphi$ are the rotation angles about the meridian plane and collected plane, respectively; $\mathbf{M}_{k}(\theta)$ is the single scattering Mueller matrix, where $\theta$ is scattering angle $(\theta \in[0, \pi])$; and $k$ is the collision time. The Mie theory is used to calculate the single scattering Mueller matrix.

After being scattered by particles in foggy environments, photons will reach our detector, allowing us to compute the DoP of forward scattered LPL and CPL, which can be expressed as [14]

$$
D o P=\frac{\sqrt{Q^{2}+U^{2}+V^{2}}}{I},
$$

which can be used to quantitatively describe the degree of incident light that persists in its initial polarization state. For the total DoP, we can calculate an average number for "I," "Q," “U," and "V." In addition, the DoPs of LPL and CPL can be calculated by DoLP $=\sqrt{Q^{2}+U^{2}} / I$ and DoCP $=V / I$, respectively.

For a better quantitative description of the transmission characteristics of circular and linear polarizations in foggy environments, we define the DoP difference as [8]

$$
\triangle D o P=D o C P-D o L P
$$

When $\triangle D o P$ is positive, the PM of circular polarization light is better, and vice versa.

\section{Results}

In general, for imaging in the complex environment, the fog is a determinant and predominant factor of visibility reduction, and the fog is formed by a large amount of water vapor molecules condense into tiny water droplets, with an average particle diameter of less than $20 \mu \mathrm{m}$. The fog can be divided into radiation fog and advection fog. Advection fog is formed when moist/warm air is advected to a colder surface, such as a maritime coastline, and is also known as marine fog; it has a particle diameter of greater than $10 \mu \mathrm{m}$, usually [25,26]. Radiation fog, also known as inland fog, is formed by the condensation of surface gas vapor due to ground radiation cooling, and its particle size is usually less than $10 \mu \mathrm{m}[23,26]$. In our work, we tried to construct monodisperse systems based on five types of foggy particles with sizes of $0.5,1.0,2.5,4$, and $5 \mu \mathrm{m}$ to simulate the transmission characteristics of LPL and CPL.

Firstly, we constructed a simple scattering environment that is composed of a single type of scattering particle with a size of $1 \mu \mathrm{m}$ and which demonstrates a visibility value of $\mathrm{V}=800 \mathrm{~m}$. Based on the MC model [15-20], we simulated the multi-spectral forward DoP evolutions of different types of polarized incidences, including those of LPL and CPL, in the constructed foggy system, as the function of the transmission length of L. The results are shown in Figure 2. In this scattering environment, we can observe that all of the DoPs decrease gradually with increasing length $(\mathrm{L})$ for all of the incident wavelengths from visible to infrared light, but the decreasing extents are different. As shown in Figure 2a, we can observe that in the visible band, the PMs of the DoCPs are better than those of the 
degree of linear polarizations (DoLPs). The PM characteristics reached optimization at $0.65 \mu \mathrm{m}$ in the visible band, and all of the DoCPs and DoLPs showed a more obvious reduction trend with increasing wavelength from 0.75 to $0.95 \mu \mathrm{m}$. As depicted in Figure 2b, in the wavelengths of near-infrared (except $2.8 \mu \mathrm{m}$ ), the PM abilities of CPL still outperformed those of LPL. In addition, at the wavelength of $1.0 \mu \mathrm{m}$, the DoLP exhibited the fastest decrease in the near-infrared band, but the evolution trends of DoCP reached optimization in the wavelength of $1.0 \mu \mathrm{m}$, which demonstrates the superior penetrating ability of CPL for this scattering system. At the same time, we can observe that the DoLP evolution trends were better than those of DoCP at the wavelength of 2.8 and $6.2 \mu \mathrm{m}$, and their values were relatively large and remained basically the same with increasing length (L), as indexed in the blue box in Figure 2b,c. From Figure 2c,d, we can observe that all DoCP evolution trends were not superior to those of the DoLPs, and these evolution trends of DoLPs and DoCPs showed a more obvious increase trend with increasing wavelength from mid-infrared to long-infrared. Put simply, the longer the wavelength is, the larger the degree of polarization is. Therefore, it can be concluded that the penetrating abilities of both CPL and LPL would be enhanced with the increase of incident wavelengths. In fact, when scanning through wavelength and size parameters, the polarization memory effect can exhibit resonant behavior, which can be attributed as the resonant behavior of the Mie scattering theory $[14,15]$.
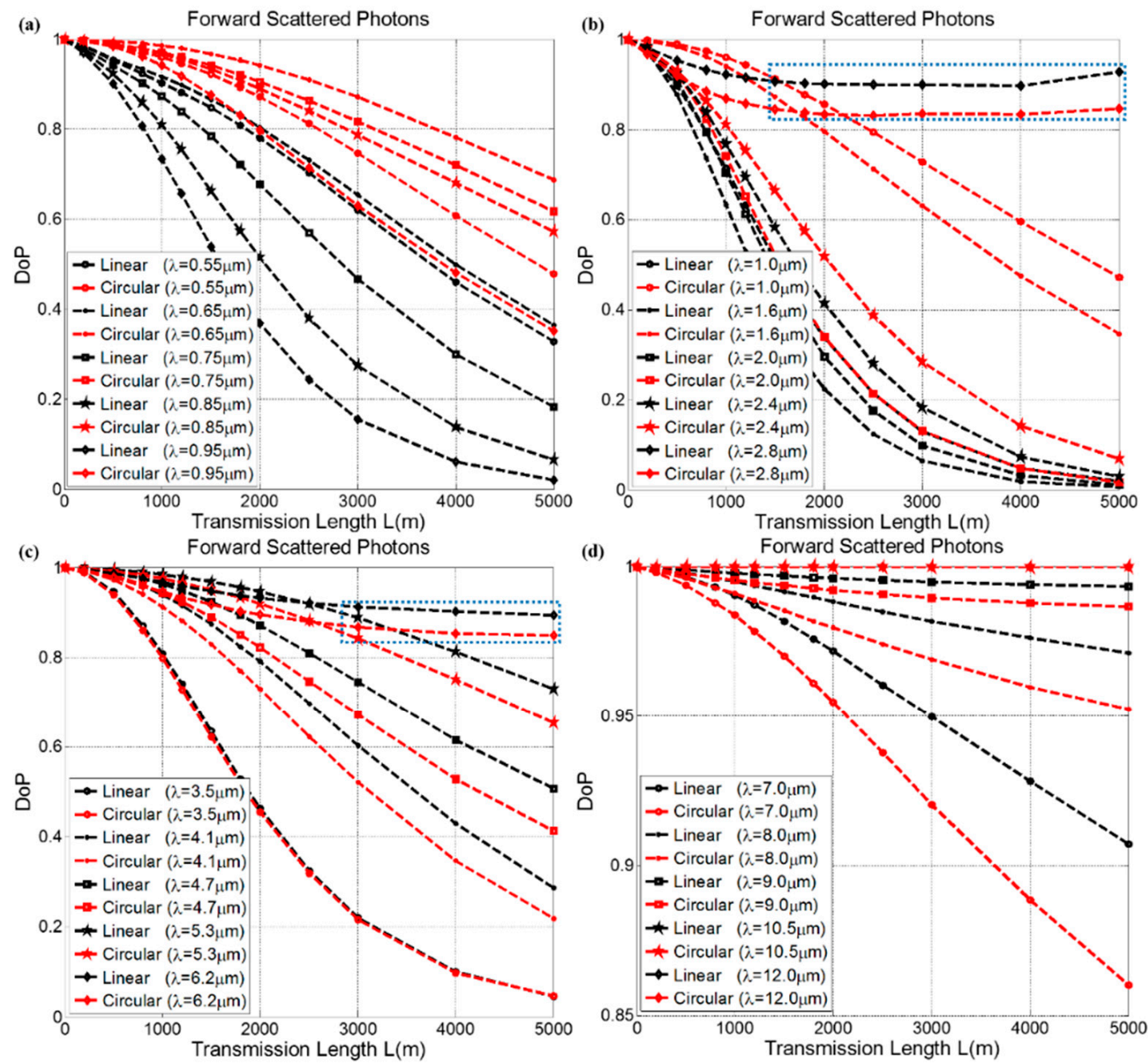

Figure 2. The transmitting degree of polarization (DoP) of linearly polarized light (LPL) and circularly polarized light (CPL) in foggy scattering environments with a particle size of $1 \mu \mathrm{m}$ (visibility value: $\mathrm{V}=800 \mathrm{~m}$ ) as the function of transmission length for different incident wavelengths: (a) Visible, (b) near-infrared, (c) mid-infrared, and (d) long-infrared band. 
For better presenting the advantages of CPL and LPL, the $\triangle D o P$ between CPL and LPL is obtained and demonstrated in Figure 3, in which the PM abilities of CPL are shown to still be better than those of LPL from the visible to near-infrared bands, except for the wavelength of $2.8 \mu \mathrm{m}$; with increasing incident wavelengths, the $\triangle D o P$ firstly increased and then decreased. The $\triangle D o P$ reached the maximum near the wavelength of $1.0 \mu \mathrm{m}$, and it showed a more obvious rise and decrease with the increase of the transmitting distance. The negative $\triangle D o P$ represents that the PM characteristics of LPL are superior to that of CPL. Meanwhile, we can observe that in the long-infrared band, the $\triangle D o P$ got closer to 0 , which demonstrates the similar penetrating abilities of the incident CPLs and LPLs in the long-infrared band.

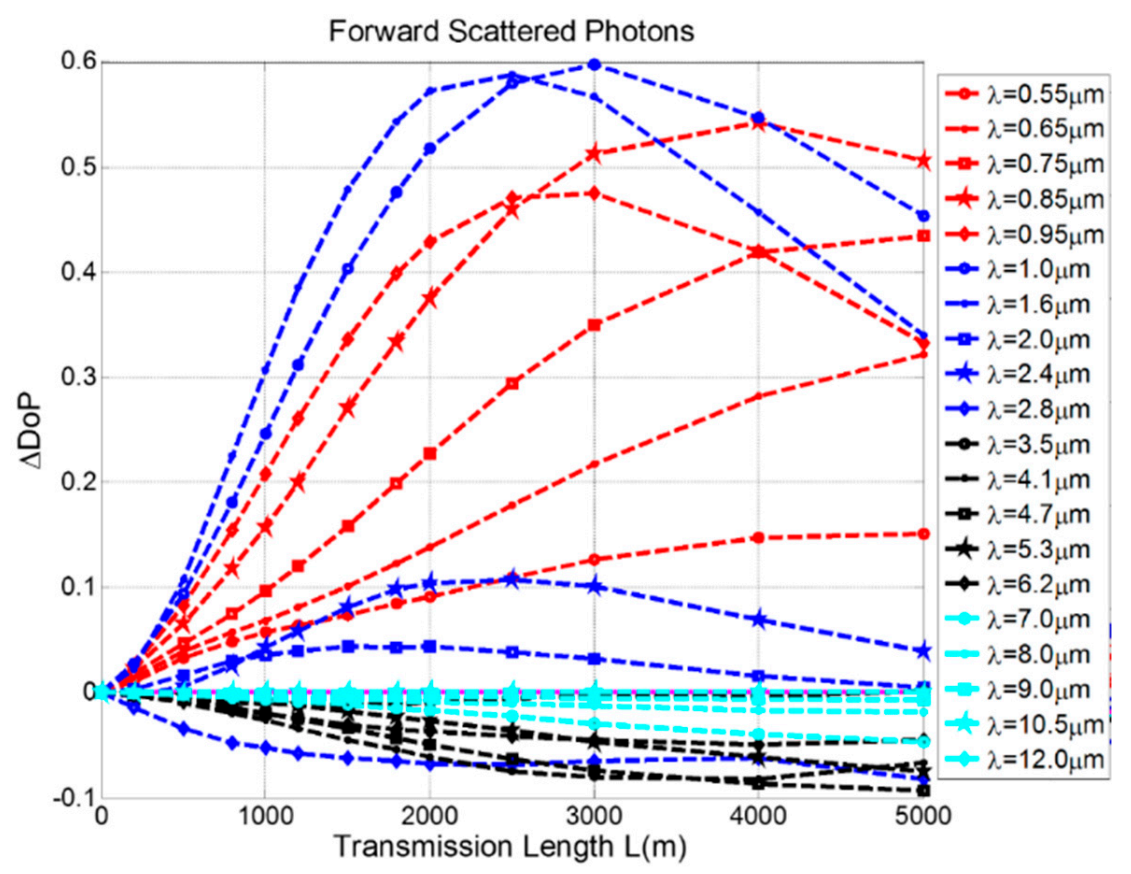

Figure 3. The transmitting DoP difference of CPL and LPL in the constructed foggy scattering environments with a particle size of $1 \mu \mathrm{m}$ as the function of transmission length for different incident wavelengths.

As shown in Figure 4, for demonstrating the scattering effects of the constructed system obviously, we calculated 2-dimension intensity distributions of the scattering lights in different detecting planes with transmission lengths of $800 \mathrm{~m}$ (Figure 4a), $1500 \mathrm{~m}$ (Figure 4b), $3000 \mathrm{~m}$ (Figure 4c), and $5000 \mathrm{~m}$ (Figure 4d). We can observe that with increasing transmission lengths, the speckles in the detecting planes will spread gradually, and the center peak will decrease accordingly. With an increasing transmission length, the collision probability between the photons and particles will be increased, so the transmitted photons will experience a larger number of scattering events. Therefore, the high-fidelity ballistic photons reaching the center of the forward detector will be gradually reduced, and more photons will be scattered to surrounding area of the central detecting planes. As a result, the center peak gradually decreases, and the side lobes are broadened. According to radial intensity distributions, all of the DoPs will decrease gradually, as shown in Figure 2. In general, the depolarization of CPL incidence is determined mostly by the helicity flipping effects of circular photons [14], but for our forward evolution model, the helicity flipping probability was relative lower. Here, the depolarization of CPL incidence can be attributed to the randomness of scattering directions (so CPL still has better PM characteristics within certain scattering events). However, the depolarization of LPL incidence is determined by both the rotations of the meridian plane and the randomness of scattering direction. As such, for the forward scattering events, after fewer scattering times (in the short transmission length), the depolarization of DoCP will be smaller compared to that of DoLP, which can be regarded as the reason of the "rising" $\Delta D o P$, as shown in Figure 3 (the transmission length is less than $3000 \mathrm{~m}$ ). 
In addition, after multiple scattering times (in the long transmission length), the depolarization of DoLP will be reduced. as shown in Figure 2a; thus the "decreasing" $\triangle D o P$ can be found in Figure 3 (the transmission length is larger than $3000 \mathrm{~m}$ ).

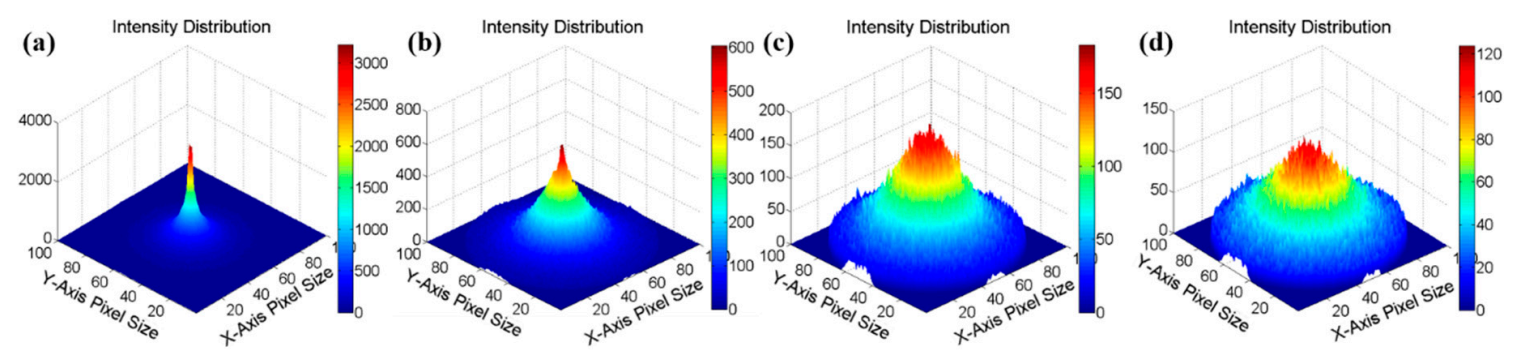

Figure 4. Two-dimensional intensity distributions of the scattering lights at the wavelength of $1 \mu \mathrm{m}$ after different transmission lengths of (a), 800 m, (b) 1500 m, (c) $3000 \mathrm{~m}$, and (d) $5000 \mathrm{~m}$.

The refractive index (RI) of scattering particles will directly determine the scattering and absorption effects in the transmission system. As shown in Figure 5a, we can observe that with increasing the incident wavelengths, the imaginary part of particles shows two obvious peaks at the wavelengths of 3 and $6 \mu \mathrm{m}$, and it also increases very fast at the long-infrared range. In these regions, the absorption cannot be negligible, and the attenuation of light is affected by both scattering and absorption effects $[27,28]$. As depicted in Figure $5 b$, with an increasing transmission length, there is a large absorption effect at these special wavelengths $(2.8 \mu \mathrm{m}, 6.2 \mu \mathrm{m}$, and $12.0 \mu \mathrm{m})$, and the normalized intensity power is already below $20 \%$ when the transmission length is larger than $3000 \mathrm{~m}$ for these high-absorption wavelengths. However, the multiple-scattered photons have a longer path through scattering systems, and when high absorption is present, multiple-scattered photons are easier to be absorbed [28,29]. As such, these photons that can reach the detector are mainly high-fidelity ballistic photons. At the same time, in these bands, the real part of the RI also has a significantly lower value (shows less scattering efficiency). Therefore, at least at these special high-absorption wavelengths, LPL and CPL have higher PM values, and the $\triangle D o P$ gets closer to 0.
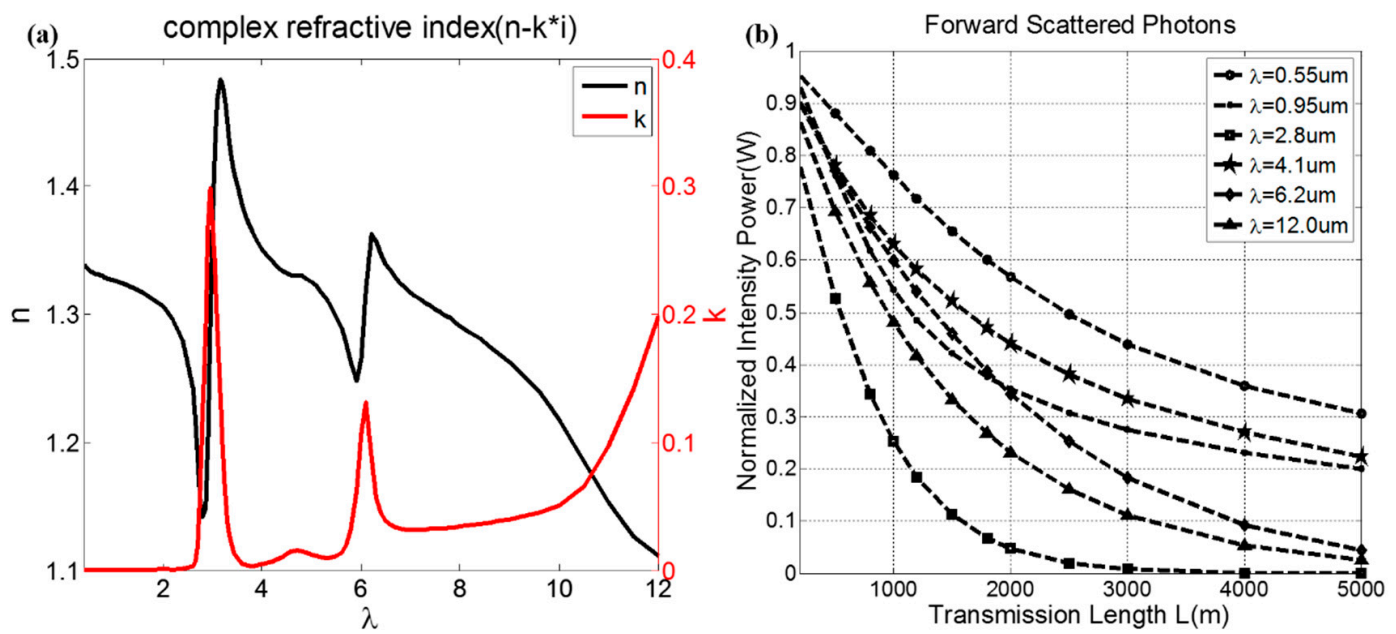

Figure 5. (a) The complex refractive index (RI) of water fog in the wavelength range of $0.4-12 \mu \mathrm{m}$. (b) The normalized intensity power of $1 \mu \mathrm{m}$ particle size as the function of transmission length from visible to infrared $(0.55 \mu \mathrm{m}, 0.95 \mu \mathrm{m}, 2.8 \mu \mathrm{m}, 4.1 \mu \mathrm{m}, 6.2 \mu \mathrm{m}$, and $12.0 \mu \mathrm{m})$.

In the above figures, we mainly explore a foggy environment with a scattering particle size of $1 \mu \mathrm{m}$. Here, we constructed monodisperse systems based on five types of foggy particles with sizes of $0.5,1.0,2.5,4$, and $5 \mu \mathrm{m}$ to simulate LPL and CPL transmission characteristics with a transmitting 
length of $2000 \mathrm{~m}$ for different incident wavelengths $(0.55,0.95,2.4,4.1$, and $5.3 \mu \mathrm{m})$. Due to the strong absorption efficiency in the long-infrared band, we did not select these bands. As shown in Figure $6 \mathrm{a}$, we can observe that in the visible band $(0.55 \mu \mathrm{m})$, when the particle size is $0.5 \mu \mathrm{m}$, the resulting DoLP has a smaller PM value (0.6772) for LPL incidence. In contrast, the resulting DoCP has a larger PM value (0.9071) for CPL incidence. In addition, from Figure 6a we can observe that, for the incident wavelength of $0.55 \mu \mathrm{m}$ with the constructed scattering systems which have the selected sizes of particles $(0.5,1.0,2.5,4$, and $5 \mu \mathrm{m}), \mathrm{CPL}$ transmission characteristics are better than those of LPL. In the near-infrared range of $0.95 \mu \mathrm{m}$, we can observe that for CPL and LPL incidences, when the particle sizes are 0.5 and $1.0 \mu \mathrm{m}$, the resulting DoPs are relatively small, especially for the particle size of $1.0 \mu \mathrm{m}$, where the resulting DoLP is only 0.3685 and the $\Delta D o P$ appears as 0.4284 , as shown in Figure $6 \mathrm{~b}$. In addition, in the near-infrared range of $0.95 \mu \mathrm{m}$, the resulting DoCPs are better than the resulting DoLPs, which can also be further proven from Figure 6b. Similarly, in the mid-infrared band of $5.3 \mu \mathrm{m}$, when the particle sizes are 4.0 and $5.0 \mu \mathrm{m}$, the DoLPs have smaller values and the DoCPs are better than the DoLPs. From above, we can also observe that when the particle sizes are similar to the incident wavelengths (the particle sizes in the system are closer to the incident wavelength), the PM characteristics of the CPL incidences demonstrate an overwhelming dominance compared to those of the LPL incidences, which can be found in the circle of the Figure $6 \mathrm{~b}$. In fact, this relationship also exists in other wavelengths (such as $2.4 \mu \mathrm{m}$ and $4.1 \mu \mathrm{m}$ ). In general, when the particle sizes are similar with the incident wavelengths, the extinction effect from the scattering system will reach to the biggest value due to the scattering effect, and the incident photons will experience most scattering events (the biggest scattering time) [9,14]. Meanwhile, compared to the better PM characteristics of CPL incidence, LPL incidences demonstrate a more severe depolarization effect. Therefore, at these regions, CPL demonstrates superior PM characteristics. From Figure 6a, we can also observe that for the scattering system with a smaller size of scattering particles (the particle size is $0.5 \mu \mathrm{m}$ ) in the wavelengths from $2.4-5.3 \mu \mathrm{m}$, the resulting DoPs remain very high (0.9810), and the resulting DoCPs and DoLPs demonstrate similar values, which have also been obviously proven in Figure $6 \mathrm{~b}$ (the $\triangle D o P$ gets closer to 0 when the particle size is $0.5 \mu \mathrm{m}$ and $1.0 \mu \mathrm{m}$ ). THis can be attributed to the smaller scattering effects of these scattering systems for the incident LPL and CPL in these wavelengths from $2.4-5.3 \mu \mathrm{m}[8]$.
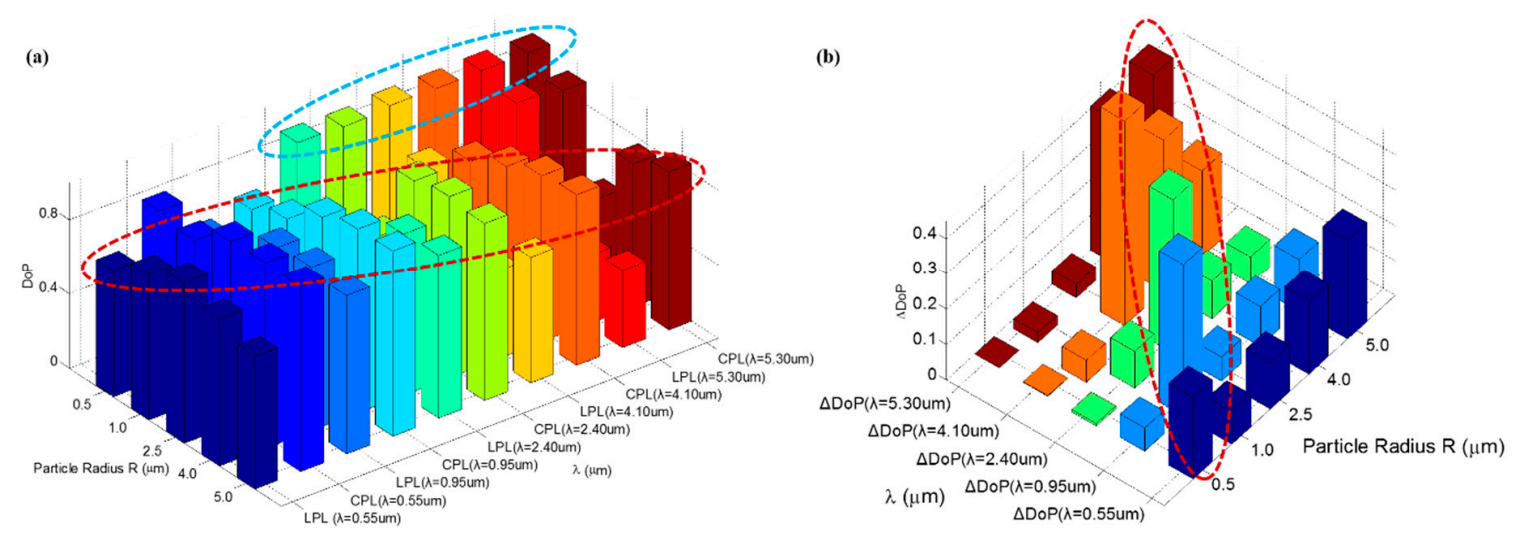

Figure 6. After transmitting $2000 \mathrm{~m}$, the resulting (a) DoP and (b) $\triangle D o P$ as the function of particle sizes of $0.5 \mu \mathrm{m}, 1.0 \mu \mathrm{m}, 2.5 \mu \mathrm{m}, 4.0 \mu \mathrm{m}$, and $5.0 \mu \mathrm{m}$ in the wavelengths of $0.55 \mu \mathrm{m}, 0.95 \mu \mathrm{m}, 2.4 \mu \mathrm{m}, 4.1 \mu \mathrm{m}$, and $5.3 \mu \mathrm{m}$, respectively.

In scattering systems, particle sizes may follow some distributions, such as Garland's measured and Kunkel's measured foggy distribution model, with a main size of $2 \mu \mathrm{m}[30,31]$, which will lead to different depolarization effects. Here, we constructed a lognormal particle spectral distribution function 
$(n(r))$, which can better demonstrate the effective radius of foggy droplets [32]. The mathematical expression can be shown as

$$
n(r)=\frac{N_{S}}{\sqrt{2 \pi} \sigma R} e^{-\frac{\left[\ln R-\ln R_{m e a n}\right]^{2}}{2 \sigma^{2}}},
$$

where $R$ is the radius of the particle size; $\sigma$ and $R_{\text {mean }}$ represent the standard deviation and mean value of the particles, respectively; and $N_{s}$ represents the total droplet concentration of per unit volume $\left(\mathrm{cm}^{-3}\right)$.

Here, in our work, we assumed that scattering systems with the mean value of the particles $R_{\text {mean }}=2.0 \mu \mathrm{m}$, and chose a six-particle-size distribution with standard deviations of $0.1 \mu \mathrm{m}, 0.3 \mu \mathrm{m}$, and $0.5 \mu \mathrm{m}$. As shown in Figure 7a, when the standard deviation was $0.1 \mu \mathrm{m}$, the six particle sizes were $1.515 \mu \mathrm{m}, 1.729 \mu \mathrm{m}, 1.944 \mu \mathrm{m}, 2.158 \mu \mathrm{m}, 2.372 \mu \mathrm{m}$, and $2.587 \mu \mathrm{m}$. With increasing the standard deviation, the particle size distribution will become wider, and the spectral density of particles will be changed accordingly. Here, to make a more prominent comparison between the PM characteristics of the incident LPL and CPL, in Figure 7d1,d2, the DoLP (Figure 7d1) lay in the range of 0.3-0.5, and the DoCP (Figure $7 \mathrm{~d} 2$ ) was in the range of $0.6-1.0$, in which the demonstrated values (starting point and ending point) in the $\mathrm{Y}$ axis were different.
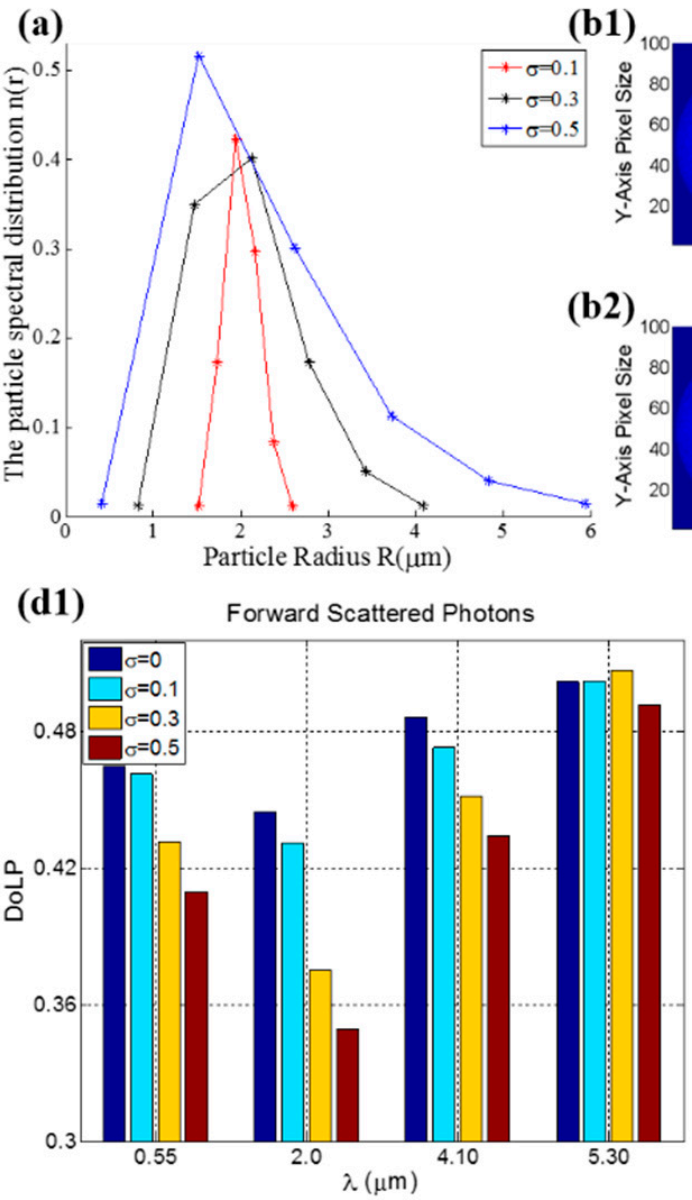

(b2)
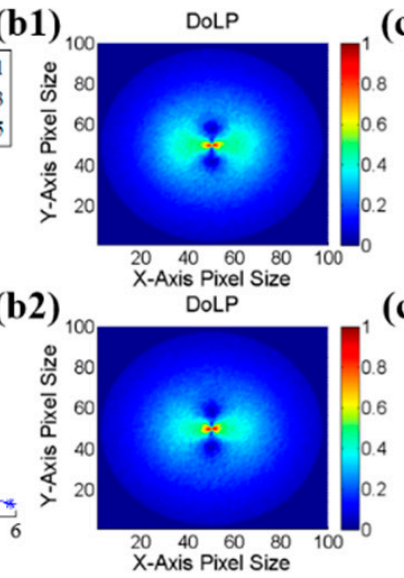

(d2)

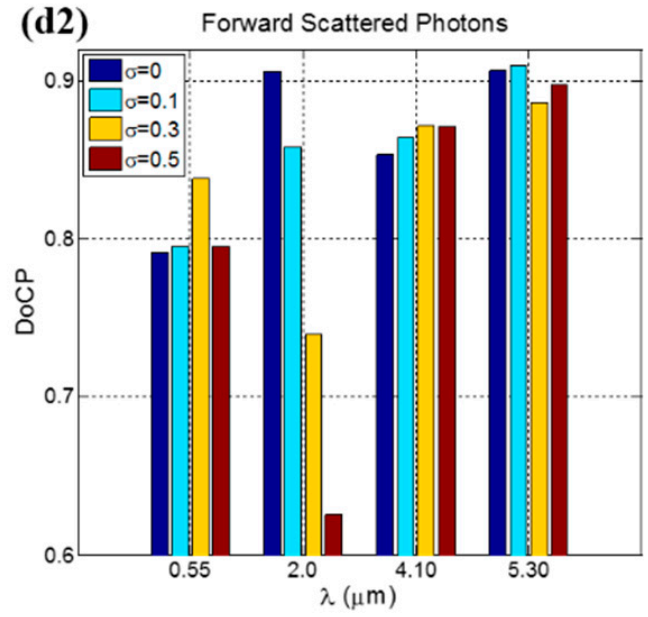

Figure 7. (a) The selected six-particle-size distribution $\left(n(r)\right.$ : Here, $\left.R_{\text {mean }}=2.0 \mu \mathrm{m}\right)$ with standard deviations of $0.1 \mu \mathrm{m}, 0.3 \mu \mathrm{m}$, and $0.5 \mu \mathrm{m}$, respectively. The resulting two-dimensional (b1) degree of linear polarization (DoLP) and (c1) degree of circular polarization (DoCP) distributions with a standard deviation of $0 \mu \mathrm{m}$. (b2) DoLP and(c2) DoCP distributions with a standard deviation of $0.5 \mu \mathrm{m}$. Also shown are the resulting (d1) DoLP and (d2) DoCP values after transmitting $2000 \mathrm{~m}$ for different incident wavelengths. 
As depicted in Figure 7d1,d2, we simulated the forward DoPs evolutions of the LPL and CPL incidences after a propagation of $2000 \mathrm{~m}$ in a six-particle-size distribution with different standard deviations for different incident wavelengths $(0.55 \mu \mathrm{m}, 2.0 \mu \mathrm{m}, 4.1 \mu \mathrm{m}$, and $5.3 \mu \mathrm{m})$. We can observe that in the scattering system with a standard deviation of $0.1 \mu \mathrm{m}$, the DoPs had almost no difference with scattering environments, with a standard deviation of 0 (single type of scattering particle with $\mathrm{r}=2.0 \mu \mathrm{m}$ ). However, the DoPs were obviously different for the system with the standard deviation of $0.5 \mu \mathrm{m}$. Especially for LPL incidence, as shown in Figure 7d1, when the standard deviation is increased, the difference of DoLPs is more obvious than that of single scattering environments. For CPL, shown in Figure 7d2, we can observe that the difference of DoCPs was not obvious compared to those of DoLPs, but there were some differences. In Figure 7b1,b2,c1,c2, we show 2-dimension DoP patterns in which we can find that, when the standard deviation is increased, the center lobe of the 2-dimension DoLP pattern becomes smaller due to the fact that, with increasing the standard deviations, particle size distribution will become wider due to the combined superposition. However, CPL has a better PM characteristic, and the differences of the 2-dimension DoCP patterns are not obvious for different size distributions. In addition, the difference of DoPs is reflected for different incident wavelengths, and the DoPs are better in the infrared band. From above, we know that a single scattering system cannot fully reflect the depolarization behavior of a poly-disperse system. The depolarization performance of polarized incidences (LPL and CPL) depends on size distributions and the sizes of scattering particles.

\section{Discussion}

We simulated the extinction and scattering efficiencies as functions of both incident wavelength and particle size by using Mie scattering theory. The scattering and extinction efficiency is shown in equation [6].

$$
\left\{\begin{array}{c}
Q_{s c a}=\frac{2}{x^{2}} \sum_{n=1}^{\infty}(2 n+1)\left(a_{n} a_{n}^{*}+b_{n} b_{n}^{*}\right) \\
Q_{\text {ext }}=\frac{2}{x^{2}} \sum_{n=1}^{\infty}(2 n+1) \operatorname{Re}\left(a_{n}+b_{n}\right)
\end{array},\right.
$$

where $a_{n}$ and $b_{n}$ can be solved by the Bessel function and $x$ is the size parameter defined as $x=2 \pi a / \lambda$. In addition, we calculated the scattering index ratio $\left(Q_{\text {ratio }}=Q_{s c a} / Q_{\text {ext }}\right)$ to better reflect the relationship between scattering and extinction efficiency. Our simulation results are presented in Figure 8.

(a)

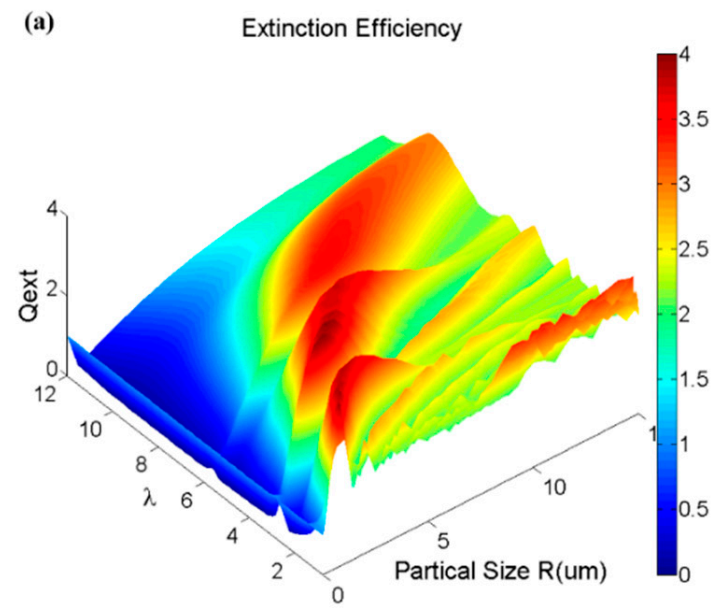

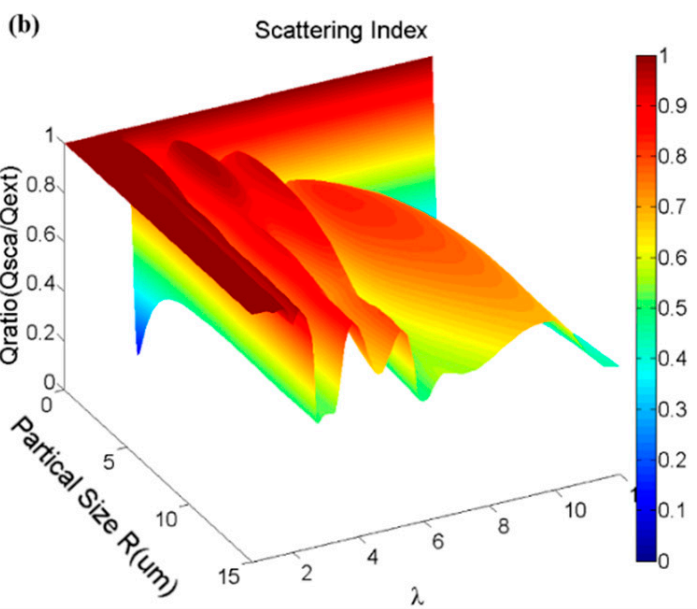

Figure 8. (a) The extinction efficiency $Q_{\text {ext }}$ and (b) scattering index ratio $Q_{\text {ratio }}$. as a function of wavelength and particle size. 
In Figure 8a, we can observe that the extinction efficiency $Q_{\text {ext }}$ has different response-peak positions in the combined coordinate systems of wavelength and particle size. These response peaks are usually located at the positions where the wavelengths and particle sizes are nearly the same values, and the extinction efficiency $Q_{\text {ext }}$ gradually becomes stable in the fluctuation with increasing particle size after reaching the peak position. In Figure $8 \mathrm{~b}$, we can see that the scattering index ratio $Q_{\text {ratio }}$ exhibits a maximum value in visible spectrum and then fluctuates as the wavelength increases. In addition, combined with Figure $5 \mathrm{~b}$, we can observe that the minimum $Q_{\text {ratio }}$ appears in the strong absorption wavelengths of $3.0 \mu \mathrm{m}, 6.0 \mu \mathrm{m}$, and a longer infrared band. As the particle size increases, the $Q_{\text {ratio }}$ shows a slow decrease. Therefore, according to the above simulation results, it is not difficult to understand the optimal and minimum cases of polarization transmission characteristics in Figures 2 and 6 . From Figures 6 and $8 b$, we can see that CPL can effectively improve its polarization transmission characteristics at positions where the scattering index ratio $Q_{\text {ratio }}$ is lower. Compared with reported works [14,33], the defined $Q_{\text {ratio }}$ can more intuitively describe the scattering characteristics of incidences with different wavelengths in scattering systems with different particle sizes. Through the relationship between scattering and total extinction, the response region between wavelength and particle size can be better found, which is conducive to analyzing the depolarization effect of particles.

\section{Conclusions}

In our work, we have investigated the transmission characteristics of different polarization states (circularly or linearly) in different foggy environments from the visible to the infrared bands. The simulation results show the scattering effect plays a major role on polarization transmission characteristics in the visible wavelength, and, when the wavelength is closer to the particle sizes in the system, the more obvious the effect on propagating polarizations. However, the DoP difference between the resulting CPL and LPL is positive at these points, which means that CPL is superior to LPL in these scattering systems. However, polarization transmission characteristics are not only affected by scattering but also by absorption in the infrared wavelength, and we can observe that CPL is not superior to LPL in the strong absorption wavelengths of $3.0 \mu \mathrm{m}, 6.0 \mu \mathrm{m}$, and the long infrared band. In addition to the above strong absorption wavelengths, when the wavelength is much larger than the particle size, both CPL and LPL have better PM characteristics, which indicates that infrared wavelength has strong penetrating characteristics in scattering systems. In addition, the distributions of particle sizes play an important role for the depolarization performances of incident light inside polydisperse systems, which means that the statistical variations of the particle sizes cannot be neglected for investigating the depolarization performances of incident light. Here, we mainly simulated spherical foggy particles in scattering environments, but, in real foggy environments, aspheric particles also exist. In future research, we will focus on the effects of non-spherical scattering foggy particles. Meanwhile, a foggy system is also highly variable, and the dynamic change factors of a foggy system should be considered in the future to further explore the problem of polarized light transmission characteristics in real foggy environments.

Author Contributions: Conceptualization, Z.G. and K.W.; methodology, F.S. and K.W.; software, T.H., F.S., and K.W.; formal analysis, X.L., K.G., F.W., Z.P., and Y.C.; investigation, R.S., K.G., Z.D., J.G., and Z.G.; writing-original draft preparation, T.H., K.W., and F.S.; writing-review and editing, T.H. and Z.G.; supervision, Z.G.; funding acquisition, K.G. and Z.G.

Funding: This research was funded by National Natural Science Foundation of China $(61775050,11804073)$, Fundamental Research Funds for the Central Universities (JZ2018HGBZ0309, JZ2018HGTB0240), and Anhui Key Laboratory of Polarization Imaging Detection Technology (2018-KFJJ-02).

Conflicts of Interest: The authors declare no conflict of interest.

\section{References}

1. Stewart, D.A.; Essenwanger, O.M. A survey of fog and related optical propagation characteristics. Rev. Geophys. 1982, 20, 481-495. [CrossRef] 
2. Ramella-Roman, J.C.; Prahl, S.A.; Jacques, S.L. Three Monte Carlo programs of polarized light transport into scattering media: Part I. Opt. Express 2005, 13, 4420-4438. [CrossRef] [PubMed]

3. Ramella-Roman, J.C.; Prahl, S.A.; Jacques, S.L. Three Monte Carlo programs of polarized light transport into scattering media: Part II. Opt. Express 2005, 13, 10392-10405. [CrossRef] [PubMed]

4. Yao, G.; Wang, L.V. Propagation of polarized light in turbid media: Simulated animation sequences. Opt. Express 2000, 7, 198-203. [CrossRef] [PubMed]

5. Xu, M.; Alfano, R.R. Circular polarization memory of light. Phys. Rev. E 2005, 72, 065601. [CrossRef] [PubMed]

6. Sun, P.; Ma, Y.; Liu, W.; Xu, C.; Sun, X. Experimentally determined characteristics of the degree of polarization of backscattered light from polystyrene sphere suspensions. J. Opt. 2013, 15, 55708. [CrossRef]

7. Schechner, Y.Y.; Narasimhan, S.G.; Nayar, S.K. Polarization-based vision through haze. Appl. Opt. 2003, 42, 511-525. [CrossRef] [PubMed]

8. Dereniak, E.L. Detection range enhancement using circularly polarized light in scattering environments for infrared wavelengths. Appl. Opt. 2015, 54, 2266.

9. van der Laan, J.D.; Wright, J.B.; Scrymgeour, D.A.; Kemme, S.A.; Dereniak, E.L. Evolution of circular and linear polarization in scattering environments. Opt. Express 2015, 23, 31874. [CrossRef] [PubMed]

10. van der Laan, J.D.; Wright, J.B.; Kemme, S.A.; Scrymgeour, D.A. Superior signal persistence of circularly polarized light in polydisperse, real-world fog environments. Appl. Opt. 2018, 57, 5464-5473. [CrossRef]

11. Hu, H.; Zhao, L.; Li, X.; Wang, H.; Yang, J.; Li, K.; Liu, T. Polarimetric image recovery in turbid media employing circularly polarized light. Opt. Express 2018, 26, 25047-25059. [CrossRef] [PubMed]

12. Li, X.; Hu, H.; Zhao, L.; Wang, H.; Han, Q.; Cheng, Z.; Liu, T. Pseudo-polarimetric Method for Dense Haze Removal. IEEE Photonics J. 2019, 11, 1-11. [CrossRef]

13. Liu, F.; Han, P.; Wei, Y.; Yang, K.; Huang, S.; Li, X.; Zhang, G.; Bai, L.; Shao, X. Deeply seeing through highly turbid water by active polarization imaging. Opt. Lett. 2018, 43, 4903-4906. [CrossRef]

14. Shen, F.; Zhang, B.; Guo, K.; Yin, Z.; Guo, Z. The Depolarization Performances of the Polarized Light in Different Scattering Media Systems. IEEE Photonics J. 2018, 10, 1-12. [CrossRef]

15. Xu, Q.; Guo, Z.; Tao, Q.; Jiao, W.; Qu, S.; Gao, J. A novel method of retrieving the polarization qubits after being transmitted in turbid media. J. Opt. 2015, 17, 35606. [CrossRef]

16. Xu, Q.; Guo, Z.; Tao, Q.; Jiao, W.; Wang, X.; Qu, S.; Gao, J. Transmitting characteristics of polarization information under seawater. Appl. Opt. 2015, 54, 6584-6588. [CrossRef] [PubMed]

17. Tao, Q.; Sun, Y.; Shen, F.; Xu, Q.; Gao, J.; Guo, Z. Active imaging with the aids of polarization retrieve in turbid media system. Opt. Commun. 2016, 359, 405-410. [CrossRef]

18. Xu, Q.; Guo, Z.; Tao, Q.; Jiao, W.; Qu, S.; Gao, J. Multi-spectral characteristics of polarization retrieve in various atmospheric conditions. Opt. Commun. 2015, 339, 167-170. [CrossRef]

19. Tao, Q.; Guo, Z.; Xu, Q.; Jiao, W.; Wang, X.; Qu, S.; Gao, J. Retrieving the polarization information for satellite-to-ground light communication. J. Opt. 2015, 17, 085701. [CrossRef]

20. Shen, F.; Wang, K.; Tao, Q.; Xu, X.; Wu, R.; Guo, K.; Zhou, H.; Yin, Z.; Guo, Z. Polarization imaging performances based on different retrieving Mueller matrixes. Opt.-Int. J. Light Electron Opt. 2018, 153, 50-57. [CrossRef]

21. Koschmieder, H. Theorie der horizontalen Sichtweite. Beitrage zur Physik der freien Atmosphare. Meteorol. Z. 1924, 12, 3353.

22. Brinkworth, B.J. Radiative transport properties of fogs. Q. J. R. Meteorol. Soc. 1964, 90, 204-207. [CrossRef]

23. Al Naboulsi, M.C.; Sizun, H.; de Fornel, F. Fog attenuation prediction for optical and infrared waves. Opt. Eng. 2004, 43, 319-330. [CrossRef]

24. Kaplan, B.; Ledanois, G.; Drévillon, B. Mueller matrix of dense polystyrene latex sphere suspensions: Measurements and Monte Carlo simulation. Appl. Opt. 2001, 40, 2769-2777. [CrossRef] [PubMed]

25. Koračin, D.; Dorman, C.E.; Lewis, J.M.; Hudson, J.G.; Wilcox, E.M.; Torregrosa, A. Marine fog: A review. Atmos. Res. 2014, 143, 142-175. [CrossRef]

26. Orgill, M.M. An Examination of the Evolution of Radiation and Advection Fogs; Science and Technology Corp.: Las Cruces, NM, USA, 1993.

27. Doicu, A.; Wriedt, T.; Eremin, Y.A. Light Scattering by Systems of Particles: Null-Field Method with Discrete Sources: Theory and Programs; Springer: Berlin/Heidelberg, Germany, 2006. 
28. Swami, M.K.; Manhas, S.; Patel, H.; Gupta, P.K. Mueller matrix measurements on absorbing turbid medium. Appl. Opt. 2010, 49, 3458-3464. [CrossRef] [PubMed]

29. Tanzid, M.; Hogan, N.J.; Sobhani, A.; Robatjazi, H.; Pediredla, A.K.; Samaniego, A.; Veeraraghavan, A.; Halas, N.J. Absorption-induced image resolution enhancement in scattering media. ACS Photonics 2016, 3, 1787-1793. [CrossRef]

30. Kunkel, B.A. Microphysical Properties of Fog at Otis AFB. Air Force Geophysics Laboratories; Air Force Systems Command, United States Air Force: Baltimore, MD, USA, 1982.

31. Garland, J.A. Some fog droplet size distributions obtained by an impaction method. Q. J. R. Meteorol. Soc. 1971, 97, 483-494. [CrossRef]

32. Yorikawa, H.; Muramatsu, S. Logarithmic normal distribution of particle size from a luminescence line-shape analysis in porous silicon. Appl. Phys. Lett. 1997, 71, 644-646. [CrossRef]

33. Macdonald, C.M.; Jacques, S.L.; Meglinski, I.V. Circular polarization memory in polydisperse scattering media. Phys. Rev. E 2015, 91, 33204. [CrossRef] [PubMed]

(C) 2019 by the authors. Licensee MDPI, Basel, Switzerland. This article is an open access article distributed under the terms and conditions of the Creative Commons Attribution (CC BY) license (http://creativecommons.org/licenses/by/4.0/). 